\title{
Éléments transposables et évolution du génome d'une espèce invasive : le cas de Drosophila simulans
}

\author{
Christian BiÉmont*, Cristina VIEIRA, Nathalie BorIE \\ Laboratoire de biométrie et biologie évolutive (UMR - CNRS 5558), \\ Université Lyon 1, 69622 Villeurbanne, France
}

Résumé - Les deux espèces jumelles $D$. melanogaster et $D$. simulans diffèrent pour leur nombre global de copies d'éléments transposables (ETs) de leur génome. $D$. simulans a moins de copies que $D$. melanogaster et le nombre de copies varie fortement entre populations naturelles. En effet, certains ETs sont absents de la plupart des populations de $D$. simulans mais présents en nombre élevé de copies dans quelques-unes. Ces données suggèrent que le génome de l'espèce $D$. simulans est en train d'être envahi par de nombreux ETs qui auraient été « mobilisés » récemment. Cette mobilisation serait reliée à la colonisation du monde par $D$. simulans. Nous donnons des arguments en faveur de cette hypothèse par une étude détaillée du nombre de copies, de la structure de ces copies et du taux de transcription du rétrotransposon 412 dans les populations des deux espèces $D$. melanogaster et $D$. simulans. Nous soutenons ainsi l'idée que la colonisation de nouveaux habitats par une espèce serait associée à une mobilisation de ses éléments transposables, ce qui conduirait à un accroissement de la taille des génomes des espèces invasives.

drosophile / éléments transposables / colonisation / populations

\begin{abstract}
Transposable elements and genome evolution of invasive species: the case of Drosophila. Drosophila simulans presents a large variation in copy number among various transposable elements (TEs) and among natural populations for a given element. Some elements are absent in most populations, except in one or two which have many copies. This suggests that some TEs are being awaken in some populations and are in the process of invading the species while it is colonizing the world. We present evidence for this hypothesis by a detailed analysis of copy number, structure of the copy sequences, and transcription rate of the retrotransposable element 412 in natural populations of the two sister species $D$. melanogaster and D. simulans. The data support the idea that colonization of new habitats by a species
\end{abstract}

* Correspondance et tirés-à-part

E-mail: biemont@biomserv.univ-lyon1.fr 
is associated with mobilization of TEs, which leads to an increase in genome size of the invading species.

\section{Drosophila / transposable elements / dynamic / colonization / natural populations}

\section{INTRODUCTION}

La taille des génomes varie considérablement entre espèces sans que cette variation soit associée à des caractéristiques évidentes de l'espèce, à sa « complexité »: c'est le paradoxe de la «valeur $\mathrm{C} »$. On sait actuellement que ces variations sont principalement le résultat de fluctuations de la quantité d'ADN répété qui, chez certaines espèces peut constituer la majorité de l'ADN [64]. Ainsi le génome humain est constitué par $95 \%$ d'ADN non codant parmi lequel $42 \%$ est constitué de séquences répétées [72]. On estime que le génome du maïs a doublé suite à une invasion de rétrotransposons [69], et qu'une amplification récente de la sous-famille $\mathrm{T}_{\mathrm{F}}$ de l'élément $\mathrm{L} 1$ a conduit à la divergence des génomes entre espèces de souris [29].

On connaît mal l'influence de cet $\mathrm{ADN}$ répété sur le génome et sur les caractéristiques de l'espèce. On pense cependant qu'une relation existe entre la taille du génome et le temps de développement, ce qui suggère un rôle de la sélection naturelle : les espèces se développant lentement pourraient supporter une quantité plus importante de cet $\mathrm{ADN}$ répété. Mais il est aussi possible que ce soit la présence d'une forte proportion de cet $\mathrm{ADN}$ qui provoque un ralentissement du développement sans que ce caractère soit sélectionné. La sélection semble pourtant être impliquée dans les clines de latitude et d'altitude pour la taille du génome observé entre divers groupes taxinomiques [39], les espèces nordiques ou d'altitude élevée présentant un plus grand génome, éventuellement avec une plus forte polyploidie, que les espèces du sud ou de faible altitude. Une telle explication est-elle valable pour les fluctuations de la taille du génome entre populations, voire entre individus ? Quelle part les éléments transposables peuvent-ils jouer dans cette explication?

On trouve les éléments transposables (ETs) à la fois chez les bactéries, les arthropodes, les plantes et les vertébrés $[42,54,66]$. Après les avoir longtemps considérés comme des parasites des génomes, on admet maintenant qu'ils ont joué un rôle important au cours de l'évolution [48,57] et qu'ils ont une influence importante dans le maintien de la variabilité génétique des populations et donc dans leur adaptation à leur environnement. Il est donc important de connaître la dynamique de tels éléments dans les génomes. Nous n'avons que peu d'information sur la manière dont les ETs envahissent les populations, surtout au cours des premières phases de cette invasion. Les exemples les plus connus sont ceux de la drosophile, Drosophila melanogaster, dont le génome a été envahi au début du siècle par les éléments $P, I$ [48], et hobo [10], tous ces éléments étant impliqués dans les phénomènes de dysgénésie des hybrides, un 
ensemble de syndromes associés à des croisements entre souches qui mobilisent ces éléments. Ces invasions impliquent que les éléments incriminés ont été mobilisés dans des populations locales ou ont été acquis par ces populations suite à un transfert horizontal. Les ETs ont alors envahi l'espèce entière par diffusion des mouches entre populations. Cinquante ans auraient suffi pour que les éléments $I$ et $P$ envahissent l'espèce entière, $D$. melanogaster, au début des années 1900. Une invasion aussi rapide et récente suggère la possibilité d'observer une invasion d'ETs dans des populations locales actuelles. Dans le présent article, nous résumons les informations récentes concernant la dynamique de divers ETs dans les populations naturelles de $D$. melanogaster et $D$. simulans, en insistant principalement sur l'élément 412 , un élément de type rétrotransposon, pour lequel de nombreuses données sont disponibles. Nous proposons que le génome de l'espèce $D$. simulans soit en train d'être envahi par plusieurs ETs à partir de quelques populations locales dans lesquelles ces ETs sont présents en nombre élevé de copies.

\section{NOMBRE DE COPIES D'ETS DANS LES POPULATIONS NATURELLES DE D. MELANOGASTER ET D. SIMULANS}

Une analyse de 34 éléments transposables dans les génomes de mouches provenant de diverses populations de $D$. melanogaster et $D$. simulans a montré que $D$. melanogaster a environ 3 fois plus de sites d'insertion d'ETs que $D$. simulans [81]. De plus, le nombre de sites est distribué selon une loi normale chez $D$. melanogaster alors qu'une large variation existe chez $D$. simulans, avec des populations à fort nombre de copies et des populations à très faible nombre, notamment pour les éléments $H M S$ beagle, blood, flea, tirant, coral, prygun, $F$, nomade et mariner. Certains de ces éléments ne sont trouvés que dans une ou deux populations et avec un nombre élevé de copies, et sont indétectables dans toutes les autres. Ces résultats suggèrent que certains ETs aient été mobilisés dans des populations locales. Un tel résultat est en contradiction avec l'idée que le faible nombre de copies d'ETs chez $D$. simulans par rapport à $D$. melanogaster serait dû à des caractéristiques particulières de D. simulans qui limiteraient le nombre de copies d'ETs dans cette espèce [49]. Cette idée était soutenue par l'observation que le nombre de sites d'insertion de l'élément $P$ dans des transformants de $D$. simulans (cette espèce ne possède pas d'élément $P$ ) obtenus par injection d'élément $P$, était plus faible que celui observé chez $D$. melanogaster $[26,60,67]$. De même les taux de transposition et d'excision de l'élément mariner de $D$. mauritiana introduit dans la lignée germinale de $D$. melanogaster, sont environ six fois plus forts que chez D. simulans [36], ce qui est en accord avec l'idée qu'un seuil critique du nombre d'ETs pourrait être spécifique de chaque espèce.

Nos données sont en faveur de l'hypothèse alternative, mais pas exclusive, que le génome de $D$. simulans n'a pas encore été envahi par la plupart des 
ETs, sauf par quelques-uns d'entre eux tels hobo, 412 et roo/B104, et en partie seulement par $I$, doc, bari-1 et helena. Il est remarquable que les éléments gypsy et $Z A M$, qui sont deux rétrovirus, ont le même nombre faible de copies chez les deux espèces $D$. melanogaster et $D$. simulans [81]. Par contre, $D$. melanogaster a été envahie il y a longtemps par la plupart de ses ETs, mais seulement très récemment par $P$ et $I[1,15]$. De même la présence de copies de l'élément helena chez seulement une ou deux populations de $D$. melanogaster, suggère que cet élément est en train d'envahir cette espèce. Nous proposons ainsi que les ETs du génome de $D$. simulans sont en train d'être « réveillés », comme ils l'ont été chez $D$. melanogaster. La conséquence d'un tel processus est que le génome de D. simulans va voir son nombre de copies d'ETs augmenter progressivement, chaque ET évoluant vers son équilibre du nombre de copies à sa propre vitesse. Ainsi, le seuil critique du nombre de copies, qui est considéré comme spécifique de l'espèce, ne doit refléter que le fait que les populations sont loin de leur valeur d'équilibre du nombre de copies pour la plupart de leurs ETs [75]. L'observation que des éléments tels bari-1 et hobo ont des nombres de copies très proches dans les deux espèces signifie alors soit que $D$. simulans ne peut pas limiter le nombre de copies de ces éléments, ou bien plus probablement que ces deux éléments ont envahi le génome de $D$. simulans il y a déjà longtemps, avant les autres ETs. On peut bien sûr argumenter et dire que bari-1 et hobo sont deux transposons, et que le génome de $D$. simulans ne contrôle pas le nombre de copies de ces éléments comme il le fait avec les rétrotransposons. Mais alors pourquoi l'élément $P$, un autre transposon, n'arrive-t-il pas à envahir le génome de $D$. simulans dans les expériences de transformation ci-dessus? Il est possible que l'état de consanguinité des lignées utilisées et leurs caractéristiques propres soient plus importants que l'espèce elle-même.

\section{LE RÉTROTRANSPOSON 412}

Parmi l'ensemble des éléments de $D$. simulans, 412 et roo/B104 présentent un nombre de copies élevé (412: 13,9 ; roo/B104:38,5), mais moins élevé cependant que chez $D$. melanogaster $(412: 28,5 ;$ roo/B104 : 67,8$)$. La distribution du nombre de copies de ces deux éléments dans les populations naturelles des deux espèces $D$. melanogaster et $D$. simulans, provenant de régions géographiques différentes du globe a montré que le nombre de copies de 412, mais pas celui de roo/B104, suit un cline Nord-Sud associé à la température minimale [78]. Ces données suggèrent que la température peut interférer avec la régulation du nombre de copies de certains ETs.

Parmi l'ensemble des populations, la population de Canberra (Australie) présente un nombre de copies très élevé pour plusieurs ETs dont 412. Ceci montre que des populations locales peuvent être envahies par des ETs soit à cause d'une petite taille efficace [20,75], soit à cause de caractéristiques particulières de ces populations qui ont conduit à la mobilisation de ces ETs [82]. 
Dans cette population de Canberra, le nombre élevé de copies de 412 n'est pas associé à un fort taux de transposition [80], ce qui suggère soit que l'élément 412 a subi une explosion temporaire de transposition, soit que la régulation du nombre de copies implique des mécanismes indépendants de ce taux [11]. Une diffusion dans la population de Canberra de mouches étrangères ne peut pas rendre compte de l'accroissement considérable du nombre de copies de 412 car aucune autre population ne montre une telle valeur ; ou bien nous devons supposer que le croisement de mouches d'origine géographique différente mobilise les ETs, ce qui serait en accord avec les forts taux de mutation observés dans les descendants de tels croisements [74,85].

Les différences de nombre de copies de 412 entre les populations de D. simulans peuvent impliquer soit des séquences particulières de l'élément soit une régulation spécifique de la transcription, qui n'existeraient pas chez D. melanogaster. Nous avons montré que bien que la structure canonique de l'élément 412 était conservée entre les deux espèces, un fort polymorphisme de structure existait dans les populations de $D$. simulans, avec beaucoup de copies réarrangées [21]. Le nombre élevé de telles copies dans le génome de D. simulans est compatible avec une récente mobilisation de l'élément 412 et avec le fait que la sélection n'a pas eu suffisamment de temps pour réduire significativement le nombre de ces copies défectives [12]. De plus, si une des forces limitant le nombre de copies consiste à réarranger ces copies, ce qui les rend inactives, une hétérogénéité fonctionnelle d'une famille d'élément transposable peut alors avoir d'importantes conséquences pour la limitation de l'accroissement du nombre de copies [14]. Il est habituel de considérer que les copies réarrangées d'ETs sont d'anciens composants du génome, le plus souvent emmagasinées dans l'hétérochromatine [16,17,24,77], bien que Sun et al. [73] aient rapporté que les centromères pouvaient comporter des copies complètes de certains éléments. Ainsi le fait que des copies complètes et réarrangées de 412 aient été trouvées à la fois dans les régions euchromatiques et hétérochromatiques du génome [21] est en accord avec l'idée d'une mobilisation récente de cet élément chez $D$. simulans, au moins plus récemment que chez $D$. melanogaster.

Pour tester l'idée que la variation du nombre de copies de l'élément 412 peut dépendre d'une régulation de la transcription [25], nous avons analysé le profil d'expression de 412 au cours des stades de développement des deux espèces $D$. melanogaster et $D$. simulans [11]. Nous avons trouvé que les profils d'expression diffèrent grandement entre populations et que globalement la quantité totale de transcrits sur l'ensemble des stades de développement est plus faible chez $D$. melanogaster que chez $D$. simulans. De plus chez cette dernière, la quantité totale de transcrits augmente avec le nombre de copies situées sur les chromosomes jusqu'à la valeur de 10-12 copies, au-delà de cette valeur la quantité de transcrits décroît avec le nombre de copies et atteint des valeurs proches de celles qui sont observées dans les populations de $D$. melanogaster. Ces résultats suggèrent que dans les populations de $D$. simulans à faible nombre de copies, le profil d'expression de l'élément 412 dépend principalement 
des séquences régulatrices de gènes de l'hôte à côté desquelles les copies sont insérées. Dans les populations à nombre élevé de copies, la transcription totale est au contraire soumise à une répression globale. L'inhibition des gènes par effet de la chromatine (chromatin-mediated silencing) ou par co-suppression (homology-dependent gene silencing), et d'autres mécanismes de répression globale sont proposés en tant que réponse du génome hôte à l'invasion des ETs $[45,58,86]$. La régulation de la transcription des ETs peut ainsi dépendre des facteurs de l'hôte, de la conformation de la chromatine ou de séquences internes à l'élément et agissant en cis $[18,35,65,70]$. Dans ce contexte, la population de Canberra est un cas particulier car elle possède un nombre de copies de 412 bien supérieur à toutes les autres populations [79], et la transcription de cet élément n'est pas soumise à une répression globale [11]. Cette population a soit transgressé les régulations cellulaires habituelles de l'élément 412, soit elle a gagné de nouvelles copies récemment suite à une mobilisation de l'élément [79]. Une mobilisation de l'élément peut être liée à l'acquisition d'une copie par transfert horizontal à partir d'une autre espèce ou bien à l'activation d'une copie inactive déjà présente dans le génome. La recombinaison entre copies homologues même situées dans l'hétérochromatine [47], et l'induction par stress peuvent être responsables des étapes initiales de l'apparition de copies « activées » susceptibles d'envahir le génome. Ainsi la recombinaison et les réarrangements entre d'anciennes copies inactives ont été proposés pour expliquer la réactivation des éléments $297[56]$ et $I[16,30]$. La mise en œuvre de tels processus est compatible avec l'observation de l'invasion du génome suite à la mobilisation soudaine de certains ETs tels copia, gypsy, $P$ et doc dans des lignées de laboratoire [6,7] et $m d g 3$ et 412 dans les populations naturelles $[9,79]$. Le génome peut alors réagir à cette invasion en produisant des copies délétées inactives [12], maintenir un nombre élevé de copies, ou même continuer d'accumuler des copies si le système de régulation n'est pas mis en place. La présence de copies réarrangées dans les populations de $D$. simulans peut alors refléter la réaction du génome à une telle invasion.

\section{L'INVASION DU GÉNOME D'UNE ESPĖCE PAR LES ETS SUIT LA COLONISATION DE NOUVEAUX HABITATS PAR L'ESPECE}

L'idée d'une invasion des génomes par les ETs pendant le processus de colonisation par une espèce est attractive car des études récentes ont montré que des populations qui colonisent des environnements nouveaux peuvent évoluer très rapidement en réponse à la sélection naturelle [63]. Les ETs pourraient participer à cette adaptation en créant une nouvelle variabilité suite à leur mobilisation et leurs insertions à des endroits nouveaux du génome, entraînant ainsi l'apparition de nouveaux variants. Il a ainsi été montré que la quantité totale d'ETs déterminée chez plusieurs populations naturelles de $D$. melanogaster, 
augmente de l'Afrique vers les autres régions du globe [8]. Cela est compatible avec une colonisation de l'ensemble du monde par cette espèce à partir de l'Afrique, lieu d'origine supposé de l'espèce [53]. Cette colonisation est alors suivie d'une mobilisation de certains ETs sans doute en tant que réponse aux stress liés aux nouveaux environnements rencontrés. Les échanges entre populations par migration/diffusion entraînent alors l'invasion du génome de l'espèce, avec comme conséquence une diminution du niveau d'activité des ETs concernés $[11,13,68]$.

Nous devrions nous intéresser aux populations naturelles d'espèces qui sont actuellement dans un processus de colonisation de nouveaux habitats et qui rencontrent de nouveaux environnements comme D. simulans $[40,53]$ qui semble beaucoup plus variable et présente une structuration de ses populations plus importante que ce qu'on pensait $[41,44,50]$ avec des effets fondateurs et des mélanges de populations [41] ; D. malerkotliana, qui s'étend rapidement vers l'Est et l'Afrique tropicale [28] ; D. buzzatii, qui a colonisé la péninsule Ibérique à partir de populations sud américaines [52]; Drosophila subobscura et D. ambigua, qui ont colonisé l'Amérique du Nord à partir de l'Europe [4] ; Chymomyza amoena, qui a été récemment introduite en Europe [55,84] ; et toutes autres espèces connues pour leur susceptibilité à l'environnement (voir [63] pour une revue et [32]) ou à des changements globaux [31].

\section{LA SENSIBILITÉ DES ETs À LEUR ENVIRONNEMENT}

La connaissance de la sensibilité des ETs aux variations de leur environnement est centrale pour comprendre leur contribution éventuelle aux capacités adaptatives des populations naturelles et à la spéciation. Ainsi l'induction de transpositions d'ETs sous l'influence de stress de l'environnement n'a été démontrée que dans quelques cas, par exemple dans les cultures de cellules ou en soumettant les plantes à des blessures ou à des stress du milieu $[43,59]$, suite à des traitements à la chaleur chez Saccharomyces cerevisiae et Candida albicans (voir [2] pour une revue), après irradiation aux UV de la bactérie Escherichia coli [33]. Il a été récemment rapporté qu'une exposition aux UV-B de pollen de maïs activait l'élément mutator initialement immobile [83]. Puisque la moitié du génome du maïs est constituée de rétrotransposons, la mobilisation d'une petite partie de ces ETs est suffisante pour induire une nouvelle variabilité génétique qui pourrait être utilisée pour une adaptation à des conditions nouvelles de l'environnement. Chez la drosophile, certains résultats soutiennent l'idée d'une mobilisation d'ETs suite à des chocs thermiques, mais d'autres données montrent l'absence d'effet de tels stress [3], bien que les chocs thermiques augmentent clairement la quantité de transcrits de l'élément copia [83]. Des relations indirectes entre facteurs de l'environnement et mobilisation d'ETs ont été mises en évidence chez la drosophile. Ainsi le taux d'excision somatique du transposon mariner augmente avec la latitude 
d'origine des populations [37] et est relié à la température [19] ; le nombre de copies du rétrotransposon 412 suit un cline de température comme montré plus haut [78]. Ces résultats peuvent s'expliquer par l'action de facteurs de régulation des taux de transposition et d'excision agissant en trans et qui dépendraient des conditions de l'environnement, mais aussi par un effet indirect des gènes de l'hôte [5], dont la fréquence varierait selon les populations et les conditions de leur environnement [22,61]. Les migrations et la colonisation d'habitats nouveaux favoriseraient ainsi la confrontation entre populations, facilitant l'échange d'ETs, comme cela a été proposé pour l'élément $P$ de la drosophile [27,38]. La formation d'hybrides entre espèces peut aussi entraîner l'activation spécifique d'ETs, comme chez l'hybride entre les deux espèces de marsupiaux australiens (Macropus eugenii et Wallabia bicolor), chez lesquels les rétrovirus endogènes ont été fortement amplifiés [62]. L'augmentation du taux de transposition d'ETs chez des hybrides interspécifiques de drosophile [34,51] suggère que la mobilisation d'ETs chez les hybrides est peut-être un processus général. Que de tels phénomènes impliquent les mécanismes moléculaires de réparation de l'ADN est une question qui mérite l'attention. Des études complémentaires sont cependant nécessaires car le taux de mutation ne semble pas augmenter chez des hybrides interspécifiques de drosophiles [23] alors qu'il augmente lors de croisements entre populations géographiquement éloignées [74].

\section{CONCLUSION}

De récentes discussions montrent que nous prenons de plus en plus conscience de l'impact des invasions d'espèces sur notre environnement [31]. Ce phénomène semble prendre une ampleur considérable dans certains milieux et la position de notre société est soit de s'en inquiéter soit de considérer que nous avons affaire à un phénomène normal ayant existé de tout temps. Malgré de nombreuses recherches, on ne sait pas prévoir les invasions, surtout on ne sait pas a priori quelles espèces ont les capacités à envahir [31,46], ni pourquoi certaines espèces ne semblent pas pouvoir envahir, et les changements génétiques associés aux invasions, qui sont sans doute complexes, sont loin d'être compris. La possibilité d'une invasion des génomes par les ETs pendant qu'une espèce est elle-même en train d'envahir de nouveaux environnements mérite d'être examinée.

Notre étude chez la drosophile suggère que les espèces colonisatrices et structurées en sous-populations temporaires voient leur génome être progressivement envahi par des copies d'éléments transposables (ETs), contrairement aux populations ancestrales et aux espèces non colonisatrices qui restent stables pour ces éléments. Une telle mobilisation des ETs, due en partie à une réponse à l'environnement (aux stress), fournirait la diversité génétique nécessaire à l'espèce pour s'adapter (les ETs sont responsables d'une part importante des mutations et leur influence au cours de l'évolution est désormais reconnue) 
et donc favoriserait la colonisation de nouveaux milieux. Il y aurait ainsi « auto entraînement » entre la diversité génétique et la colonisation de nouveaux milieux. La conséquence d'un tel phénomène est l'augmentation de la taille des génomes des colonisateurs, une explication partielle du paradoxe de la valeur C $[64,71,76]$ entre populations. La rançon d'un tel processus serait une augmentation, au moins temporaire, de la charge en mutations associées aux ETs, donc du fardeau génétique des individus. Les recherches futures devraient inclure les formes intégrées des rétrovirus dont la quantité dans les génomes pourrait augmenter en même temps que les autres éléments transposables suite à une augmentation de la connectivité entre populations hôtes.

\section{REMERCIEMENTS}

Nous remercions les lecteurs pour leurs remarques. L'ensemble du travail présenté dans ce manuscrit a été soutenu par le BRG, le CNRS (programme génome, GDR 2157) et l'Association pour la recherche sur le cancer.

\section{RÉFÉRENCES}

[1] Anxolabéhère D., Kidwell M.G., Periquet G., Molecular characteristics of diverse populations are consistent with the hypothesis of a recent invasion of Drosophila melanogaster by mobile P elements, Mol. Biol. Evol. 5 (1988) 252-269.

[2] Arnault C., Dufournel I., Genome and stresses: Reactions against aggressions, behavior of transposable elements, Genetica 93 (1994) 149-160.

[3] Arnault C., Lœvenbruck C., Biémont C., Transposable element mobilization is not induced by heat shocks in Drosophila melanogaster, Naturwissenschaften 84 (1997) 410-414.

[4] Beckenback A.T., Prévosti A., Colonization of North America by the european species, Drosophila subobscura and D. ambigua, Amer. Nat. 115 (1986) 10-18.

[5] Bhadra U., Pal-Bhadra M., Birchler J.A., A trans-acting modifier causing extensive overexpression of genes in Drosophila melanogaster, Mol. Gen. Genet. 254 (1997) 621-634.

[6] Biémont C., Population genetics of transposable DNA elements. A Drosophila point of view, Genetica 86 (1992) 67-84.

[7] Biémont C., Dynamic equilibrium between insertion and excision of P elements in highly inbred lines from an M' strain of Drosophila melanogaster, J. Mol. Evol. 39 (1994) 466-472.

[8] Biémont C., Vieira C., Borie N., Lepetit D., Transposable elements and genome evolution: The case of Drosophila simulans, Genetica 107 (2000) 113-120.

[9] Biémont C., Vieira C., Hoogland C., Cizeron G., Lœvenbruck C., Arnault C., Carante J.P., Maintenance of transposable element copy number in natural populations of Drosophila melanogaster and D. simulans, Genetica 100 (1997) $161-166$. 
[10] Bonnivard E., Bazin C., Denis B., Higuet D., A scenario for the hobo transposable element invasion, deduced from the structure of natural populations of Drosophila melanogaster using tandm TPE repeats, Genet. Res. Camb. 76 (2000) 217-226.

[11] Borie N., Lœvenbruck C., Biémont C., Developmental expression of 412 retrotransposon in natural populations of D. melanogaster and D. simulans, Genet. Res. Camb.(2000) (sous presse).

[12] Brookfield J.F.Y., Models of repression of transposition in P-M hybrid sdysgenesis by $\mathrm{P}$ cytotype and by zygotically encoded repressor proteins, Genetics 128 (1991) 471-486.

[13] Brookfield J.F.Y., Models of the spread of non-autonomous selfish transposable elements when transposition and fitness are coupled, Genet. Res. 67 (1996) 199-209.

[14] Brookfield J.F.Y., Badge R.M., Population genetics models of transposable elements, Genetica 100 (1997) 281-294.

[15] Bucheton A., Paro R., Sang H.M., Pélisson A., Finnegan D.J., The molecular basis of IR hybrid dysgenesis in D. melanogaster: Identification, cloning and properties of the I factor, Cell 38 (1984) 153-163.

[16] Bucheton A., Vaury C., Chaboissier M.C., Abad P., Pélisson A., Simonelig M., I elements and the Drosophila genome, Genetica 86 (1992) 175-190.

[17] Busseau I., Chaboissier M.C., Pélisson A., Bucheton A., I factors in Drosophila melanogaster: Transposition under control, Genetica 93 (1994) 101-116.

[18] Cavarec L., Heidmann T., The Drosophila copia retrotransposon contains binding sites for transcriptional regulation by homeoproteins, Nucleic Acids Res. 21 (1993) 5041-5049.

[19] Chakrani F., Capy P., David J.R., Developmental temperature and somatic excision rate of mariner transposable element in three natural populations of Drosophila simulans, Genet. Select. Evol. 25 (1993) 121-132.

[20] Charlesworth B., Charlesworth D., The population dynamics of transposable elements, Genet. Res. Camb. 42 (1983) 1-27.

[21] Cizeron G., Biémont C., Polymorphism in structure of the retrotransposable element 412 in Drosophila simulans and D. melanogaster populations, Gene 232 (1999) 183-190.

[22] Costa R., Peixoto A.A., Barbujani G., Kyriacou C.P., A latitudinal cline in a Drosophila clock gene, Proc. R. Soc. Lond. [Biol.] 250 (1992) 43-49.

[23] Coyne J.A., Mutation rates in hybrids between sibling species of Drosophila, Heredity 63 (1989) $155-162$.

[24] Crozatier M., Vaury C., Busseau I., Pélisson A., Bucheton A., Structure and genomic organization of I elements involved in I-R hybrid dysgenesis in Drosophila melanogaster, Nucleic Acids Res. 16 (1988) 9199-9213.

[25] Csink A.K., Mcdonald J.F., Copia expression is variable among natural populations of Drosophila, Genetics 126 (1990) 375-385.

[26] Daniels S.B., Clark S.H., Kidwell M.G., Chovnick A., Genetic transformation of Drosophila melanogaster with an autonomous P element: phenotypic and molecular analyses of long-established transformed lines, Genetics 115 (1987) 711-23. 
[27] Daniels S.B., Peterson K.R., Strausbaugh L.D., Kidwell M.G., Chovnick A., Evidence for horizontal transmission of the $\mathrm{P}$ transposable element between drosophila species, Genetics 124 (1990) 339-355.

[28] David J.R., Tsacas L., Cosmopolitan, subcosmopolitan and widespread species: Different strategies within the Drosophilid family (Diptera), C. R. Soc. Biogéo. 57 (1981) 11-26.

[29] DeBerardinis R.J., Goodier J.L., Ostertag E.M., Kazazian H.H., Rapid amplification of a retrotransposon subfamily is evolving the mouse genome, Nature Genet. 20 (1998) 288-290.

[30] Dimitri P., Constitutive heterochromatin and transposable elements in Drosophila melanogaster, Genetica 100 (1997) 85-93.

[31] Duke J.S., Mooney H.A., Does global change increase the success of biological invaders?, Trends Ecol. Evolut. 14 (1999) 135-139.

[32] Duvernell D.D., Turner B.J., Variation and divergence of Death Valley pupfish populations at retrotransposon-defined loci, Mol. Biol. Evol. 16 (1999) 363-371.

[33] Eichenbaum Z., Livneh Z., UV light induces IS 10 transposition in Escherichia coli, Genetics 149 (1998) 1173-1181.

[34] Evgen'ev M.B., Yenikolopov G.N., Peunova N.I., Ilyin Y.V., Transposition of mobile genetic elements in interspecific hybrids of Drosophila, Chromosoma 85 (1982) 375-386.

[35] Flavell A.J., Ruby S.W., Toole J.J., Roberts B.E., Rubin G.M., Translation and developmental regulation of RNA encoded by the eukaryotic transposable element copia, Proc. Natl. Acad. Sci. USA 77 (1980) 7107-7111.

[36] Garza D., Medhora M., Koga A., Hartl D.L., Introduction of the transposable element mariner into the germline of Drosophila melanogaster, Genetics 128 (1991) 303-10.

[37] Giraud T., Capy P., Somatic activity of the mariner transposable element in natural populations of Drosophila simulans, Proc. R. Soc. Lond. [Biol.] 263 (1996) 1481-1486.

[38] Good A.G., Meister G.A., Brock H.W., Grigliatti T.A., Hickey D.A., Rapid spread of transposable $\mathrm{P}$ elements in experimental populations of Drosophila melanogaster, Genetics 122 (1989) 387-396.

[39] Gregory T.R., Hebert P.D., The modulation of DNA content: Proximate causes and ultimate consequences, Genome Res. 9 (1999) 317-24.

[40] Hale L.R., Singh R.S., Contrasting patterns of genetic structure and evolutionary history as revealed by mitochondrial DNA and nuclear gene-enzyme variation between Drosophila melanogaster and Drosophila simulans, J. Genet. 70 (1991) 79-90.

[41] Hamblin M.T., Veuille M., Population structure among african and derived populations of Drosophila simulans: Evidence for ancient subdivision and recent admixture, Genetics 153 (1999) 305-317.

[42] Heslop-Harrison J.S., Brandes A., Taketa S., Schmidt T., Vershinin A.V., Alkhimova E.G., Kamm A., Doudrick R.L., Schwarzacher T., Katsiotis A., Kubis S., Kumar A., Pearce S.R., Flavell A.J., Harrison G.E., The chromosomal distribution of Ty1-copia group retrotransposable elements in higher plants and their implications for genome evolution, Genetica 100 (1997) 197-204. 
[43] Hirochika H., Sugimoto K., Otsuki Y., Tsugawa H., Kanda M, Retrotransposons of rice involved in mutations induced by tissue culture, Proc. Natl. Acad. Sci. USA 9 (1996) 7783-7788.

[44] Irvin S.D., Wetterstrand K.A., Hutter C.M., Aquadro C.F., Genetic variation and differentiation at microsatellite loci in Drosophila simulans: Evidence for founder effects in new world populations, Genetics 150 (1998) 777-790.

[45] Jensen S., Gassama M.-P., Heidmann T., Taming of transposable elements by homology-dependent gene silencing, Nature 21 (1999) 209-212.

[46] Kareiva P., Developing a predictive ecology for non-indigenous species and ecological invasions, Ecology 77 (1996) 1651-1652.

[47] Ke N., Voytas D.F., cDNA of the yeast retrotransposon Ty5 preferentially recombines with substrates in silent chromatin, Mol. Cell. Biol. 19 (1999) 484-494.

[48] Kidwell M.G., Lisch D.R., Transposable elements as sources of variation in animals and plants, Proc. Natl. Acad. Sci. USA 94 (1997) 7704-7711.

[49] Kimura K., Kidwell M.G., Differences in P element population dynamics between the sibling species Drosophila melanogaster and D. simulans, Genet. Res. 63 (1994) 27-38.

[50] Labate J.A., Biermann C.H., Eanes W.F., Nucleotide variation at the runt locus in Drosophila melanogaster and Drosophila simulans, Mol. Biol. Evol. 16 (1999) 724-731.

[51] Labrador M., Farré M., Utzet F., Fontdevilla A., Interspecific hybridization increases transposition rates of Osvado, Mol. Biol. Evol. 16 (1999) 931-937.

[52] Labrador M., Seleme M.C., Fontdevila A., The evolutionary history of Drosophila buzzatii. XXXIV. The distribution of the retrotransposon Osvaldo in original and colonizing populations, Mol. Biol. Evol. 15 (1998) 1532-1547.

[53] Lachaise D., Cariou M., David J.R., Lemeunier F., Tsacas L., Ashburner M., Historical biogeography of the Drosophila melanogaster species subgroup, Evol. Biol. 22 (1988) 159-227.

[54] Löwer R., Löwer J., Kurth R., The viruses in all of us: Characteristics and biological significance of human endogenous retrovirus sequences, Proc. Natl. Acad. Sci. USA 93 (1996) 5177-5184.

[55] Maca J., Bächli G., On the distribution of Chymomyza amoena (Loew), a species recently introduced into Europe, Bull. Soc. Ent. Suisse 67 (1994) 183-188.

[56] Martin G., Wiernasz D., Schedl P., Evolution of Drosophila repetitive-dispersed DNA, J. Mol. Evol. 19 (1983) 203-213.

[57] McDonald J.F., Transposable elements: Possible catalists of organismic evolution, Trends Ecol. Evol. 10 (1995) 123-126.

[58] McDonald J.F., Transposable elements, gene silencing and macroevolution, Trends Ecol. Evol. 13 (1998) 98-95.

[59] Mhiri C., Morel J.B., Vernettes S., Casacuberta J.M., Lucas E., Grandbastien M.A. The promoter of the tobacco Tnt1 retrotransposon is induced by wounding and by abiotic stress, Plant Mol. Biol. 33 (1997) 257-266.

[60] Montchamp-Moreau C., Hybrid dysgenesis in P-transformed lines of Drosophila simulans, Evolution 44 (1990) 194-203.

[61] Oakeshott J.G., Gibson J.B., Anderson P.R., Knibb W.R., Anderson D.G., Chambers G.K., Alcohol dehydrogenase and glycerol-3-phosphate dehydrogenase clines in Drosophila melanogaster on different continents, Evolution 36 (1992) 86-96. 
[62] O’Neill R.J.W., O'Neill M.J., Graves J.A.M., Undermethylation associated with retroelement activation and chromosome remodelling in an interspecific mammalian hybrid, Nature 393 (1998) 68-72.

[63] Orr M.R., Smith T.B., Ecology and speciation, Trends Ecol. Evol. 13 (1998) 502-506.

[64] Pagel M., Johnstone R.A., Variation across species in the size of the nuclear genome supports the junk-DNA explanation for the C-value paradox, Proc. $\mathrm{R}$. Soc. Lond. [Biol.] 249 (1992) 119-124.

[65] Parkhurst S.M., Corces V.G., Developmental expression of Drosophila melanogaster retrovirus-like transposable elements, EMBO J. 6 (1987) 419-24.

[66] Robertson H.M., Lampe D.J., Distribution of transposable elements in arthropods, Annu. Rev. Entomol. 40 (1995) 333-357.

[67] Rubin G.M., Spradling A.C., Genetic transformation of Drosophila with transposable element vectors, Science 218 (1982) 348-53.

[68] Russell A.L., Woodruff R.C., The genetics and evolution of the mariner transposable element in Drosophila simulans: Worldwide distribution and experimental population dynamics, Genetica 105 (1999) 149-164.

[69] SanMiguel P., Gaut B.S., Tikhonov A., Nakajima Y., Bennetzen J.L., The paleontology of intergene retrotransposons of maize, Nature Genet. 2 (1998) 43-45.

[70] Scherer G., Telford J., Baldari C., Pirrotta V., Isolation of cloned genes differentially expressed at early and late stages of Drosophila embryonic development, Dev. Biol. 86 (1981) 438-447.

[71] Sherwood S.W., Patton J., Genome evolution in Pocket Gophers (genus Thomomys). II. Variation in cellular DNA content, Chromosoma 85 (1982) 163-179.

[72] Smit F.A., Interspersed repeats and other mementos of transposable elements in mammalian genomes, Curr. Opin. Genetics. Dev. 9 (1999) 657-663.

[73] Sun X., Wahlstrom J., Karpen G., Molecular structure of a functional Drosophila centromere, Cell 91 (1997) 1007-1019.

[74] Thompson Jr., J.N., Woodruff R.C., Increased mutation in crosses between geographically separated strains of Drosophila melanogaster, Proc. Natl. Acad. Sci. USA 77 (1980) 1059-1062.

[75] Tsitrone A., Charles S., Biémont C., Dynamics of transposable elements under the selection model, Genet. Res. 74 (1999) 159-164.

[76] Uozu S., Ikehashi H., Ohmido N., Ohtsubo H., Ohtsubo E., Fukui K., Repetitive sequences: Cause for variation in genome size and chromosome morphology in the genus Oryza, Plant Mol. Biol. 35 (1997) 791-799.

[77] Vaury C., Chaboissier M.C., Drake M.E., Lajoinie O., Dastugue B., Pélisson A., The $d o c$ transposable element in D. melanogaster and D. simulans: Genomic distribution and transcription, Genetica 93 (1994) 117-124.

[78] Vieira C., Aubry P., Lepetit D., Biémont C., A temperature cline in copy number for 412 but not roo/B104 retrotransposons in populations of Drosophila simulans, Proc. R. Soc. Lond. (Biol.) 265 (1998) 1-5.

[79] Vieira C., Biémont C., Geographical variation in insertion site number of retrotransposon 412 in Drosophila simulans, J. Mol. Evol. 42 (1996) 443-451.

[80] Vieira C., Biémont C., Transposition rate of the 412 retrotransposable element is independent of copy number in natural populations of Drosophila simulans, Mol. Biol. Evol. 14 (1997) 185-188. 
[81] Vieira C., Lepetit D., Dumont S., Biémont C., Wake up of transposable elements following Drosophila simulans worldwide colonization, Mol. Biol. Evol. 16 (1999) 1251-1255.

[82] Vieira C., Piganeau G., Biémont C., High copy numbers of multiple transposable elements in an australian population of D. simulans, Genet. Res. 76 (2000) 117-119.

[83] Walbot V., UV-B damage amplified by transposons in maize, Nature 397 (1998) 398-399.

[84] Withers P., Allemand R., Chymomyza amoena (Loew), drosophile nouvelle pour la France (Diptera Drosophilidae), Bull. Mens. Soc. linn. Lyon 67 (1998) 159-160.

[85] Woodruff R.C., Thompson J.N., Lyman R.F., Intraspecific hybridization and the release of mutator activity, Nature 278 (1979) 277-279.

[86] Yoder J.A., Walsh C., Bestor T.H., Cytosine methylation and the ecology of intragenomic parasites, Trends Genet. 13 (1997) 335-340. 